\title{
ARTICLE Endophilin A2 regulates calcium-activated chloride channel activity via selective autophagy-mediated TMEM16A degradation
}

\author{
Can-zhao Liu ${ }^{1}$, Fei-ya Li ${ }^{1,2,3}$, Xiao-fei Lv ${ }^{1}$, Ming-ming Ma ${ }^{1}$, Xiang-yu Li ${ }^{1}$, Cai-xia Lin ${ }^{1}$, Guan-lei Wang ${ }^{1}$ and Yong-yuan Guan ${ }^{1}$
}

\begin{abstract}
TMEM16 $\mathrm{A} \mathrm{Ca}^{2+}$-activated chloride channel (CaCC) plays an essential role in vascular homeostasis. In this study we investigated the molecular mechanisms underlying downregulation of TMEM16A CaCC activity during hypertension. In cultured basilar artery smooth muscle cells (BASMCs) isolated from $2 \mathrm{k} 2 \mathrm{c}$ renohypertesive rats, treatment with angiotensin II $(0.125-1 \mu \mathrm{M})$ dosedependently increased endophilin A2 levels and decreased TMEM16A expression. Similar phenomenon was observed in basilar artery isolated from $2 \mathrm{k} 2 \mathrm{c}$ rats. We then used whole-cell recording to examine whether endophilin A2 could regulate TMEM16A CaCC activity in BASMCs and found that knockdown of endophilin A2 significantly enhanced CaCC activity, whereas overexpression of endophilin A2 produced the opposite effect. Overexpression of endophilin A2 did not affect the TMEM16A mRNA level, but markedly decreased TMEM16A protein level in BASMCs by inducing ubiquitination and autophagy of TMEM16A. Ubiquitin-binding receptor p62 (SQSTM1) could bind to ubiquitinated TMEM16A and resulted in a process of TMEM16A proteolysis in autophagosome/lysosome. These data provide new insights into the regulation of TMEM16A CaCC activity by endophilin A2 in BASMCs, which partly explains the mechanism of angiotensin-II-induced TMEM16A inhibition during hypertension-induced vascular remodeling.
\end{abstract}

Keywords: TMEM16A; $\mathrm{Ca}^{2+}$-activated chloride channel; basilar smooth muscle cells; angiotensin II; endophilin A2; ubiquitination; autophagy; hypertension; vascular remodeling

Acta Pharmacologica Sinica (2020) 41:208-217; https://doi.org/10.1038/s41401-019-0298-5

\section{INTRODUCTION}

TMEM16A/Ano1 is the molecular identity of the $\mathrm{Ca}^{2+}$-activated chloride channel $(\mathrm{CaCC})$ in eukaryotic cells [1-4]. The TMEM16Amediated $\mathrm{CaCC}$ has been reported to regulate a variety of physiological functions, including neuronal excitability, nociception, transepithelial ion transport, and gastrointestinal peristalsis [5-9]. Our recent study found that CaCC activity in the cardiovascular system, as well as TMEM16A expression level, was decreased in basilar artery smooth muscle cells (BASMCs) isolated from twokidney two-clip $(2 \mathrm{k} 2 \mathrm{c})$ renohypertensive rats, possibly caused by a high concentration of angiotensin II during hypertension [10]. Although diverse physiological and pathological functions have been described, regulatory mechanisms of the TMEM16A channel have rarely been reported. Calmodulin, one of the many modulators, has been identified as a factor contributing to the $\mathrm{Ca}^{2+}$ dependence of TMEM16A channel activity [11-13]. Recently, another intriguing study reported that secreted calcium-activated chloride channel regulator 1 could modulate TMEM16A-dependent calcium-activated chloride currents by stabilizing TMEM16A on the cell surface in HEK293T cells [14], which gives rise to new insight into seeking proper adaptor proteins required for TMEM16A CaCC modulation. A recent proteomics study identified several endogenous proteins implicated in protein trafficking, folding, and stability that interact with TMEM16A, including SNARE complexes such as syntaxins and syntaxin-binding proteins, as well as ezrin-radixin-moesin scaffolding proteins [15]. These studies reveal the importance of the regulation of TMEM16A distribution and stability on CaCC activity. Identification of appropriate modulators in different tissues is crucial to precisely understand TMEM16A functions.

Endophilin A2 is a member of the endophilin protein family that is widely expressed in eukaryotic cells and profoundly exists in smooth muscle cells compared with the other family members [16]. Endophilins are composed of two conserved functional domains, including a sizeable N-terminal BAR (Bin-amphiphysin-Rvs) domain, which can induce and stabilize membrane curvature upon dimerization, and a C-terminal SH3 (Src homology 3) domain, which can recognize and bind to proline-rich domains (PRDs). There are other undiscovered domains that mediate essential functions as well [17-19]. Evidence has shown that endophilin A2 plays a vital role in regulating clathrin-independent endocytosis and clathrindependent endocytosis due to its unique protein structure [20]. In addition, amino acid residues 200-219 mediate the direct interaction of the endophilin- $\mathrm{Ca}^{2+}$ channel complex, whereas E264, which is flanked with a PRD, is required to form a binding

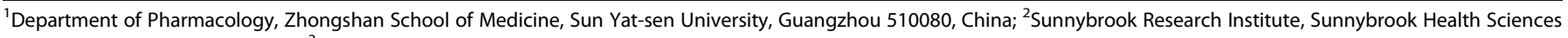
Centre, Toronto, ON, Canada and ${ }^{3}$ Department of Laboratory Medicine and Pathobiology, University of Toronto, Toronto, ON, Canada

Correspondence: Can-zhao Liu (liucanzhao0521@163.com) or Yong-yuan Guan (guanyy@mail.sysu.edu.cn)

These authors contributed equally: Can-zhao Liu, Fei-ya Li

Received: 21 April 2019 Accepted: 6 August 2019

Published online: 4 September 2019 
site for $\mathrm{Ca}^{2+}$ and can affect the conformation of the endophilin A2 protein in a $\mathrm{Ca}^{2+}$-dependent manner, eventually modulating the formation of the endophilin $\mathrm{A} 2-\mathrm{Ca}^{2+}$ channel complex [21]. Based on multiple related functions of endophilin $A 2$, we tested the hypothesis that endophilin A2 could regulate TMEM16A CaCC activity. In this study, we identified endophilin A2 as a vital regulator involved in TMEM16A CaCC activity in smooth muscle cells.

\section{MATERIALS AND METHODS}

Animals

For primary BASMC isolation, 4-week-old Sprague-Dawley rats were used. Endophilin A2 transgenic mice were generated as described previously [22]. All experiments were approved by and conducted in accordance with the ethical guidelines of the Sun Yat-Sen University Animal Experimentation Committee and were in complete compliance with the National Institutes of Health Guide for the Care and Use of Laboratory Animals. Efforts were made to minimize any pain or discomfort, and the minimum number of animals was used.

\section{Cell culture}

Rat BASMCs were isolated and cultured from rat basilar arteries using the explant method as previously described [23, 24]. Briefly, 4-week-old male Sprague-Dawley rats $(150 \sim 180 \mathrm{~g})$ were anesthetized with pentobarbital sodium (60 $\mathrm{mg} / \mathrm{kg}$, intraperitoneally). The basilar arteries were collected immediately and immersed in Krebs solution containing $10^{5} \mathrm{U} / \mathrm{L}$ penicillin and $100 \mathrm{mg} / \mathrm{L}$ streptomycin. After the connective tissue was cleaned, the basilar arteries were cut into small pieces $\sim 0.5 \mathrm{~mm}$ long and the vessel segments were placed on the surface of the culture dish. The dish was then incubated in Dulbecco's modified Essential medium/F-12 supplemented with $20 \%$ fetal calf serum at $37{ }^{\circ} \mathrm{C}$ and $5 \% \mathrm{CO}_{2}$. Passages 8-12 of the cultured BASMCs were used for experiments.

\section{Adenoviral infection}

Ad-Endophilin A2 was designed and produced by Sunbio Medical Biotechnology (Shanghai, China). Briefly, cultured BASMCs at $50 \%$ confluence were infected with adenovirus encoding endophilin A2 for $4 \mathrm{~h}$ and then the cells were washed with phosphatebuffered saline (PBS) and incubated in fresh culture medium before experimentation.

\section{Plasmid transfection}

Plasmids were transfected with Lipofectamine 2000 reagent (Invitrogen $^{\mathrm{TM}}$, Life Technologies, Grand Island, NY, USA). Briefly, plasmids were mixed with Lipofectamine 2000 in OPTI-MEMRI medium (Invitrogen ${ }^{\mathrm{TM}}$, Life Technologies) and incubated with cells according to the manufacturer's instructions. Six hours later, the cells were rinsed with PBS and incubated in fresh culture medium for $42 \mathrm{~h}$ before cell lysate collection.

\section{siRNA transfection}

The sequences of small interfering RNA (siRNA) (Supplementary Table S1) against rat endophilin A2 and p62 were synthesized (Invitrogen, Life Technologies). Briefly, siRNA was diluted in $100 \mu \mathrm{L}$ culture medium and mixed with $12 \mu \mathrm{L}$ HiPerFect reagent (Qiagen, Valencia, CA, USA) for $20 \mathrm{~min}$ at room temperature. The mixture was then added to the cells and incubated for $3 \mathrm{~h}$ in serum-free medium (the final siRNA concentration was $40 \mathrm{nM}$ ). Finally, the cells were washed once with PBS and incubated in standard culture medium for another $45 \mathrm{~h}$. The siRNA efficiency was detected by Western blotting (Supplementary Fig. S1).

\section{Reverse-transcription PCR}

Briefly, total RNA was isolated using Trizol reagent (Invitrogen ${ }^{\mathrm{TM}}$, Life Technologies) according to the manufacturer's instructions. One picogram of total RNA was reverse-transcribed in a total volume of
$30 \mu \mathrm{L}$ on a Thermal Cycler (Thermo Scientific, Waltham, MA, USA) according to the instructions of the One-step RT-PCR Kit (Qiagen, Valencia, CA, USA). Reactants underwent reverse transcription $\left(50^{\circ} \mathrm{C}\right.$, $30 \mathrm{~min})$, an initial denaturation $\left(95^{\circ} \mathrm{C}, 15 \mathrm{~min}\right), 35$ amplification cycles $\left(94^{\circ} \mathrm{C}, 45 \mathrm{~s} ; 55^{\circ} \mathrm{C}, 45 \mathrm{~s} ; 72^{\circ} \mathrm{C}, 60 \mathrm{~s}\right)$, and a final extension $\left(72{ }^{\circ} \mathrm{C}\right.$, $10 \mathrm{~min})$. The specific primers are listed in Supplementary Table S2.

Western blotting analysis

Cells or tissues were lysed with RIPA lysis buffer containing a protease inhibitor cocktail (Merck, Germany). Protein was separated with $10 \%$ SDS-polyacrylamide gel electrophoresis (PAGE) and transformed to a polyvinylidene difluoride (PVDF) membrane. After blocking with 3\%-5\% milk at room temperature for $1 \mathrm{~h}$, the membrane was incubated with primary antibody (Supplementary Table S3) at $4{ }^{\circ} \mathrm{C}$ overnight and then incubated for $1 \mathrm{~h}$ with secondary antibody at room temperature. Final detection was carried out with a chemiluminescent horseradish peroxidase substrate (Millipore) by using a BIO-RAD molecular imager, ChemiDoc XRS + . The densities of the target bands were determined by Image Lab 4.0.

\section{Co-immunoprecipitation}

Cell lysates were incubated with precoupled antibodies bound to protein $\mathrm{G}$ beads overnight at $4{ }^{\circ} \mathrm{C}$. The beads were centrifuged and washed, and then boiled in protein SDS sample buffer. Samples were resolved on $10 \%$ SDS-PAGE gels and transferred to PVDF membranes. The bound proteins were determined by immunoblotting with the indicated antibodies.

\section{Electrophysiology}

The $\mathrm{Ca}^{2+}$-activated $\mathrm{Cl}^{-}$channel currents were recorded with an Axopatch 200B Amplifier (Axon Instruments, Foster City, CA, USA) using the whole-cell recording technique at room temperature. Patch pipettes were made from borosilicate capillary tubes with a Sutter P-97 horizontal puller (Sutter Instrument, Novato, CA, USA). The resistance of the pipettes was 3-6 $\mathrm{M} \Omega$ after filling with pipette solution. The currents were elicited with voltage steps from -100 $\mathrm{mV}$ to $+100 \mathrm{mV}$ in a $+20 \mathrm{mV}$ increment for $250 \mathrm{~ms}$ with an interval of $5 \mathrm{~s}$ from a holding potential of $-50 \mathrm{mV}$. Currents were filtered at $2 \mathrm{kHz}$ and sampled at $5 \mathrm{kHz}$ using pCLAMP8.0 software (Axon Instruments). The extracellular solution contained (mM): NMDG-Cl 125, KCl 5, $\mathrm{CaCl}_{2} 1.5, \mathrm{MgSO}_{4} 1, \mathrm{HEPES} 10$, glucose 10 and $\mathrm{pH}$ was adjusted to 7.4 with NMDG. The pipette solution contained (mM): $\mathrm{CsCl} 130, \mathrm{Mg} \cdot \mathrm{ATP} 1, \mathrm{MgCl}_{2}$ 1.2, HEPES 10, EGTA 2, $\mathrm{CaCl}_{2} 1.639$ and $\mathrm{pH}$ was adjusted to 7.4 with $\mathrm{CsOH}$. The intracellular $\mathrm{Ca}^{2+}$ concentration was $260 \mathrm{nM}$.

\section{Immunofluorescence}

BASMCs were seeded onto poly-lysine-treated dishes and transfected with pEGFP-LC3 and red fluorescent protein (RFP) endophilin A2. After $24 \mathrm{~h}$ of incubation, the cells were fixed with $4 \%$ paraformaldehyde. After permeabilization with blocking buffer (PBS containing 5\% normal donkey serum, 1\% bovine serum albumin), the cells were incubated overnight in blocking buffer at $4{ }^{\circ} \mathrm{C}$ with the indicated antibodies (Supplementary Table S3). Subsequently, the dishes were rinsed in washing buffer (PBS with $0.2 \% \mathrm{TX}-100$ ) and incubated for $2 \mathrm{~h}$ at room temperature with fluorescently conjugated secondary antibodies and Hoechst diluted in blocking buffer. Pictures were acquired on a confocal microscope (Fluoview FV1000, Olympus).

\section{Statistical analysis}

All statistical analyses were performed using GraphPad Prism 5 (GraphPad Software, La Jolla, CA, USA). All data are expressed as the mean $\pm S D$. Statistical differences were assessed with the unpaired two-tailed Student's $t$-test for two experimental groups and one-way and two-way analysis of variance for more than two groups. $P<0.05$ was considered statistically significant. 


\section{RESULTS}

Endophilin A2 is upregulated during hypertension

We previously identified TMEM16A as the molecular identity of the CaCC in smooth muscle cells and found that CaCC activity was associated with a high concentration of angiotensin II in the basilar artery of the $2 \mathrm{k} 2 \mathrm{c}$ renohypertensive rat model. In this study, by employing BASMCs isolated from a $2 \mathrm{k} 2 \mathrm{c}$ renohypertensive rat model, we found that angiotensin II could increase endophilin A2 levels and decrease TMEM16A expression in a dose-dependent manner (Fig. 1a). Similar results were also found in basilar arteries isolated from the $2 \mathrm{k} 2 \mathrm{c}$ model. As shown in Fig. $1 \mathrm{~b}$, a significant increase in endophilin A2 was observed in 4-week-old $2 \mathrm{k} 2 \mathrm{c}$ renohypertensive rat basilar arteries compared with Sham control arteries. There have been reports on the association between endophilin A2 and calcium regulation, as well as the association between endophilin A2 and TMEM16A expression during hypertension, and we therefore wanted to test the effects of endophilin A2 on TMEM16A CaCC activity.

Endophilin A2 modulates the activity of CaCCs

To determine whether endophilin A2 could regulate TMEM16A CaCC activity, we carried out experiments to record $I_{\text {Cacc. }}$ BASMCs were transfected with endophilin A2 fluorescent-labeled siRNA or nonspecific, scrambled RNA. $I_{\text {Cacc }}$ of the fluorescent-labeled cells was recorded by whole-cell patch clamp electrophysiology. In the presence of $260 \mathrm{nM}$ intracellular $\mathrm{Ca}^{2+}$ and a physiological concentration of extracellular $\mathrm{Cl}^{-}$, a robust outward rectifying current was recorded in the endophilin A2 siRNA group compared with the nonspecific, scrambled RNA or untransfected group (Fig. 2a). By contrast, overexpression of endophilin A2 significantly decreased $\mathrm{CaCC}$ activity according to the recording of $I_{\mathrm{CaCC}}$ of green fluorescent protein (GFP)-expressing cells infected with endophilin A2-GFP adenovirus or GFP control (Fig. 2b). Similar results were found in BASMCs freshly isolated from endophilin A2 transgenic mice [22] and their littermate controls (Fig. 3c). These data suggest that endophilin A2 can modulate $\mathrm{CaCC}$ activity in smooth muscle cells.

Endophilin $\mathrm{A} 2$ regulates the expression of TMEM16A protein Next, to evaluate how endophilin A2 modulates TMEM16A CaCC activity in smooth muscle cells, we determined the mRNA and protein levels of TMEM16A with overexpression or knockdown of endophilin A2. The quantitative reverse-transcription PCR (qRT$P C R)$ results showed that there was no significant effect on TMEM16A mRNA level by adenovirus-mediated endophilin A2 overexpression or endophilin A2 knockdown (Fig. 3a). However, the Western blotting results revealed that the TMEM16A protein level was significantly decreased following adenovirus-mediated overexpression of endophilin $\mathrm{A} 2$, whereas knockdown of endophilin A2 increased the protein level of TMEM16A (Fig. 3b). The expression of TMEM16A was also reduced in the basilar arteries isolated from endophilin A2 transgenic mice (Fig. 3d). Furthermore, the calcium-activated $\mathrm{Cl}^{-}$current was decreased in BASMCs freshly isolated from endophilin A2 transgenic mice compared with WT mice (Fig. 3c). Together, these data implicate that endophilin A2 plays a causal role in modulating TMEM16A CaCC activity and modulates TMEM16A protein expression by regulating TMEM16A posttranslational modification rather than transcriptional regulation.

Upregulation of TMEM16A ubiquitination by endophilin A2 as a prerequisite for selective autophagy-mediated degradation In our study, we showed that angiotensin II induced a decrease in TMEM16A protein in BASMCs. To elucidate how angiotensin II regulates TMEM16A protein expression, we detected the effects of TMEM16A ubiquitination following angiotensin II stimulation. The results showed that TMEM16A ubiquitination was significantly increased following stimulation of angiotensin II, whereas the expression level of TMEM16A was decreased (Fig. 4a). Similar results were found in the basilar arteries isolated from the hypertension model in vivo (Fig. 4b).

Considering the significant upregulation of endophilin A2 in the basilar arteries isolated from the hypertension model, we hypothesized that overexpression of endophilin $A 2$ could reduce TMEM16A protein expression by modulating TMEM16A ubiquitination, which would further lead to downregulation of $\mathrm{CaCC}$ activation. By immunoprecipitating TMEM16A with ubiquitin, we found that overexpression of endophilin $A 2$ resulted in robust polyubiquitination of TMEM16A (Fig. 4c). To identify the exact degradation mechanism by which endophilin $A 2$ regulated TMEM16A protein homeostasis, we used concanamycin $A$, a specific inhibitor of V-type ATPases that can inhibit lysosome activity, to evaluate its effect on TMEM16A protein levels. As shown in Fig. $5 a$, the suppression of TMEM16A by endophilin A2 was significantly reversed by concanamycin $A$. Similar results were found by recruiting bafilomycin $A 1$, another lysosome inhibitor that is involved in the maturation of autophagic vacuoles, as demonstrated by inhibiting fusion between autophagosomes and lysosomes (Fig. 5b). However, no significant difference could be detected when the cells were pretreated with or without the proteasome inhibitor MG132 (Supplementary Fig. S2). These data suggest that the modification of TMEM16A ubiquitination tends to be delivered to the lysosome and eventually degrades in the autophagosome/lysosome.

Endophilin A2 regulates autophagy in BASMCs

To confirm whether endophilin A2 was a regulator of autophagy in BASMCs and whether autophagosomes/lysosomes represent a major method by which TMEM16A is degraded, we detected LC3 I/II conversion and GFP-LC3 puncta, which are both hallmarks of autophagosome activation. Significantly increased expression levels of LC3 and evoked conversion of LC3 I to LC3 II were observed with adenovirus-mediated endophilin A2 overexpression (Fig. 5c). Obvious GFP-LC3 puncta were found in SMC cells that were cotransfected with an RFP-endophilin A2 plasmid and a GFP-LC3 plasmid (Fig. 5d). We also detected autophagy flux levels to confirm this observation by using bafilomycin $A 1$ and the results revealed that overexpressing endophilin A2 dramatically increased autophagy flux compared with the LacZ control (Supplementary Fig. S3). These data suggest the critical role of endophilin $A 2$ in regulating autophagosome/lysosome activity, which is associated with TMEM16A degradation.

P62(SQSTM1) is required as a bridge coupling ubiquitinated TMEM16A and autophagosomes/lysosomes

To further understand how ubiquitinated TMEM16A is delivered to autophagosomes/lysosomes, we tested the interaction between TMEM16A and p62 (SQSTM1), a ubiquitin-binding receptor that can bind $\mathrm{Ub}$ and autophagosome-associated Ub-like (UBL) proteins such as LC3, which mediates wharfing of ubiquitinated protein to the autophagosome. Co-immunoprecipitation experiments showed that p62 (SQSTM1) could bind to both endophilin $A 2$ and TMEM16A, implying the potential essential roles of p62 (SQSTM1) in crosslinking ubiquitinated TMEM16A and autophagosome-associated UBL proteins (Fig. 6a, b). To shed light on the significance of p62(SQSTM1) in the degradation of ubiquitinated TMEM16A, we took advantage of p62 (SQSTM1) siRNA to knock down endogenous p62. Similar to concanamycin A and bafilomycin A1, the disruption of p62 (SQSTM1) caused a significant reversal of the degradation of TMEM16A mediated by endophilin A2 (Fig. 6c). In addition, the immunofluorescence results showed that puncta positive for $L C 3$, as well as p62 with TMEM16A, were increased in adenovirus-mediated endophilin A2overexpressing cells (Supplementary Fig. S4). Altogether, these data suggest that ubiquitinated TMEM16A modulated by endophilin $A 2$ is recognized by $\mathrm{p} 62$ (SQSTM1) and is finally delivered to 
a

\begin{tabular}{|c|c|c|}
\hline ant 0.125 & 0.25 & 0.5 \\
\hline
\end{tabular}

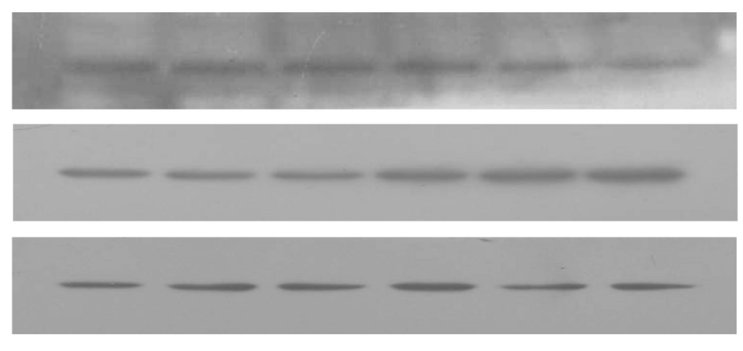

\section{TMEM16A}

\section{Endophilin A2}

GAPDH
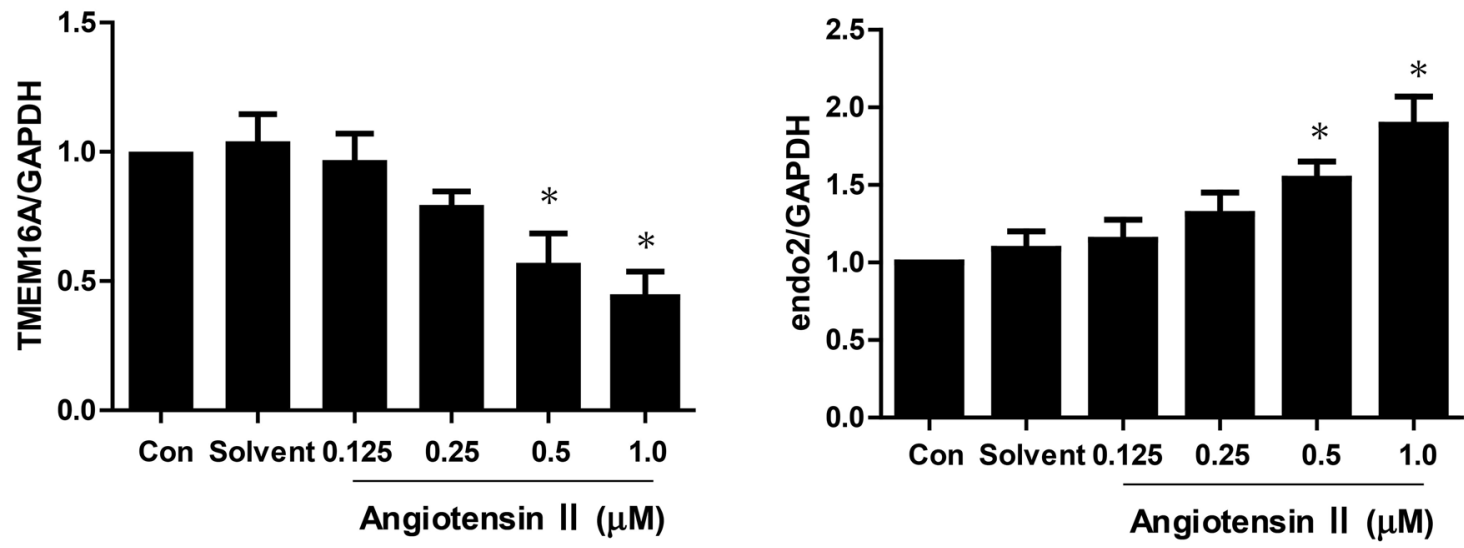

b Sham Hyper Sham Hyper

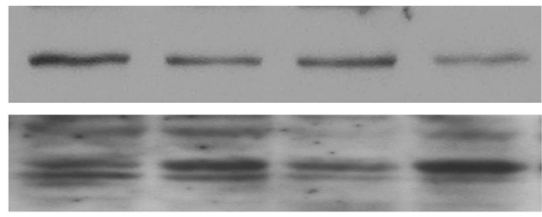

\section{TMEM16A}

\section{Endophilin A2}

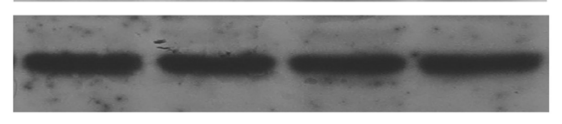

\section{GAPDH}
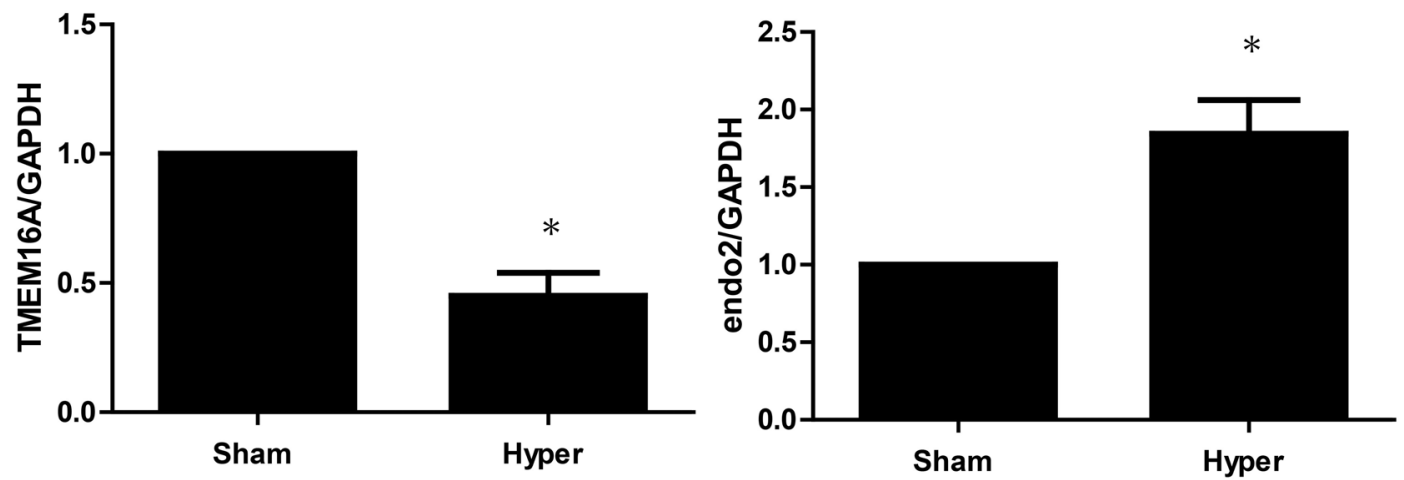

Fig. 1 Expression of Endophilin A2 and TMEM16A in hypertensive rat basilar artery and BASMCs in response to Angiotensin II stimuli. a BASMCs were incubated with Angiotensin II at concentrations indicated above for $48 \mathrm{~h}$; TMEM16A and Endophilin A2 expression were detected by Western blotting. $n=6 ;{ }^{*} P<0.05$ vs. Control (Con). b Two-kidney two-clip (2k2c) renovascular stroke-prone hypertensive rats were operated 4 weeks later; TMEM16A and Endophilin A2 expression in sham operation (Sham and hypertension (Hyper) basilar artery were detected by Western blotting). $n=6 ;{ }^{*} P<0.05$ vs. Sham 
a

a1
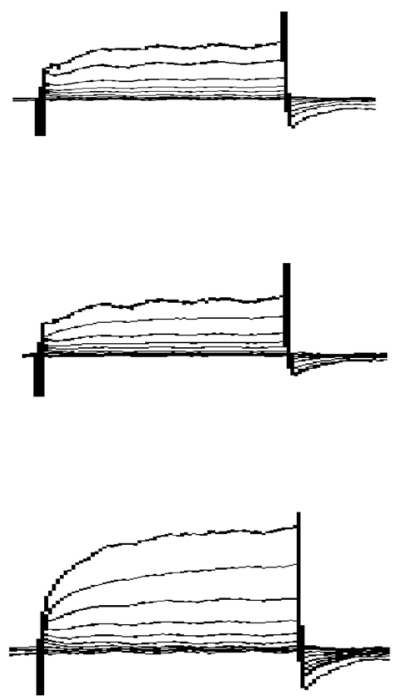

b b1
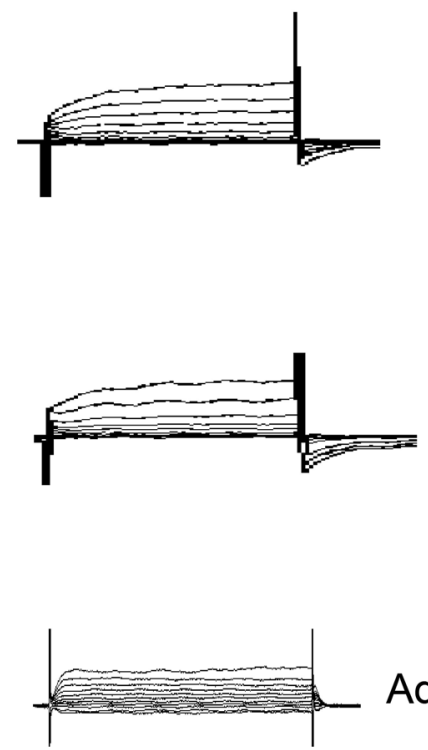

Ad-GFP-Endo2

$0.1 \mathrm{nA}$

Neg

Con

Endo2

SiRNA

Con

Ad-GFP

a2

a3
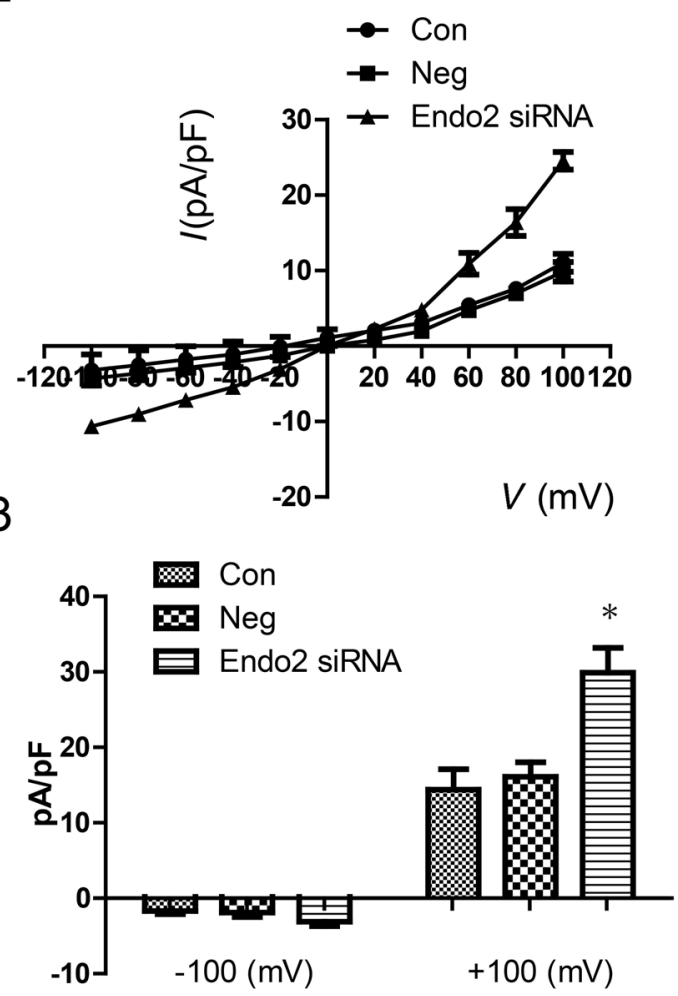

b2

b3
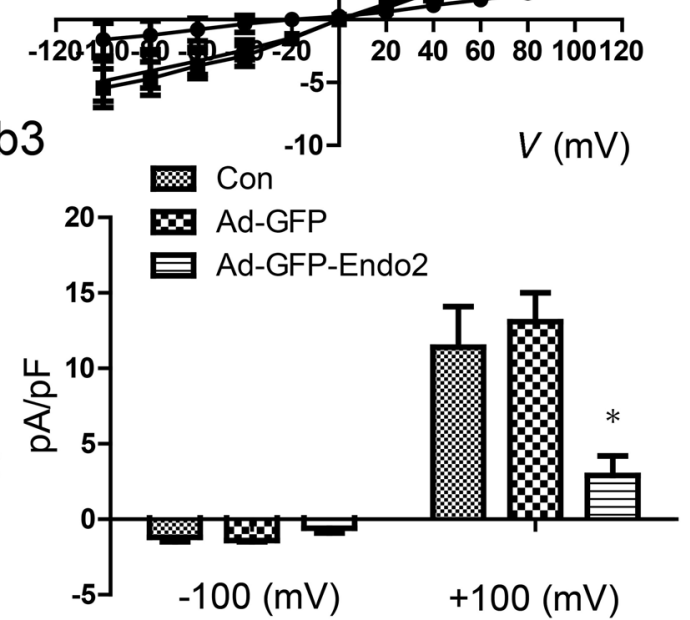

Fig. 2 Endophilin $\mathrm{A} 2$ modulates the activity of calcium-activated $\mathrm{Cl}^{-}$channel. a Representative traces of calcium-activated $\mathrm{Cl}^{-}$current (a1), I- $V$ curves (a2), and mean current densities measured at $\pm 100 \mathrm{mV}$ in Control (Con), nonspecific, scrambled RNA (Neg), Endophilin A2 siRNA knockdown (Endo2 siRNA), which were evoked by $260 \mathrm{nM}\left[\mathrm{Ca}^{2+}\right]_{i} . n=8,{ }^{*} P<0.05 \mathrm{vs}$. Con and Neg group. b Representative traces of calciumactivated $\mathrm{Cl}^{-}$current (b1), I-V curves (b2), and mean current densities measured at $\pm 100 \mathrm{mV}$ (b3) in control, GFP adenovirus (Ad-GFP), and GFP-Endophilin A2 adenovirus (Ad-GFP-Endo2) groups, which were evoked by $260 \mathrm{nM}\left[\mathrm{Ca}^{2+}\right]_{\mathrm{i}} \cdot n=8,{ }^{*} P<0.05$ vs. Con and Ad-GFP group 
a

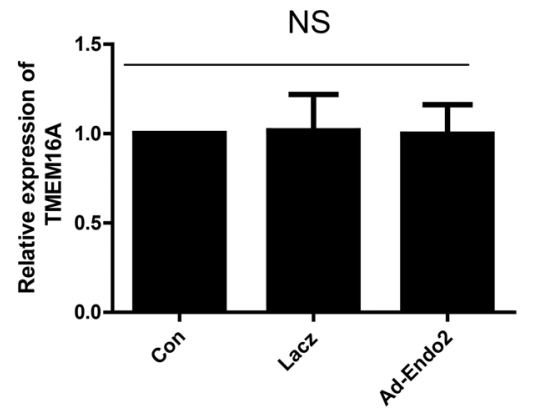

b Ad-Endo2 (MOI)

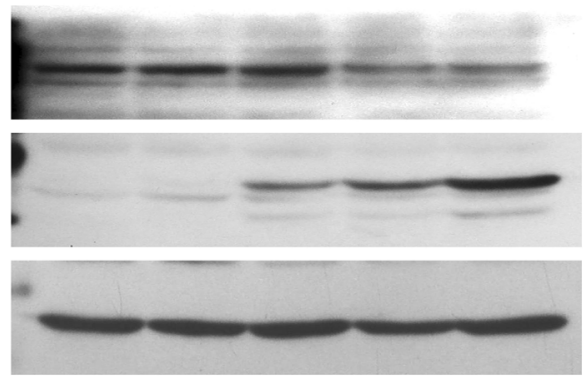

TMEM16A

$114 \mathrm{kDa}$

Endophilin A2

$47 \mathrm{kDa}$

GAPDH

$36 \mathrm{kDa}$

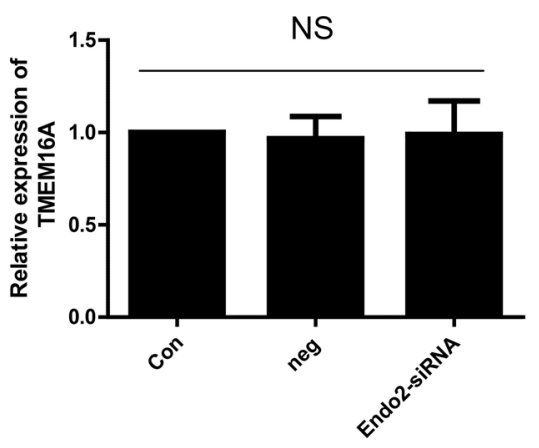

Endo2 siRNA (nM)

Con Neg $20 \quad 40 \quad 80$

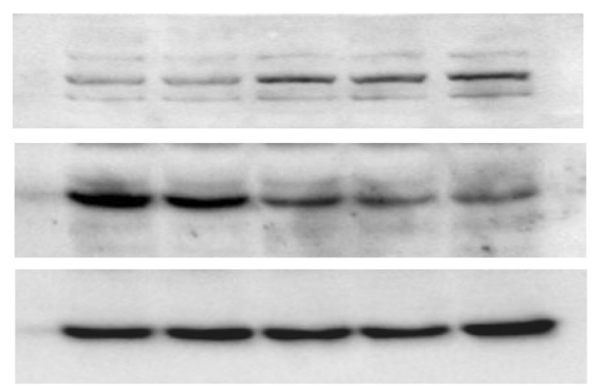

TMEM16A

$114 \mathrm{kDa}$

Endophilin A2

$47 \mathrm{kDa}$

GAPDH

$36 \mathrm{kDa}$
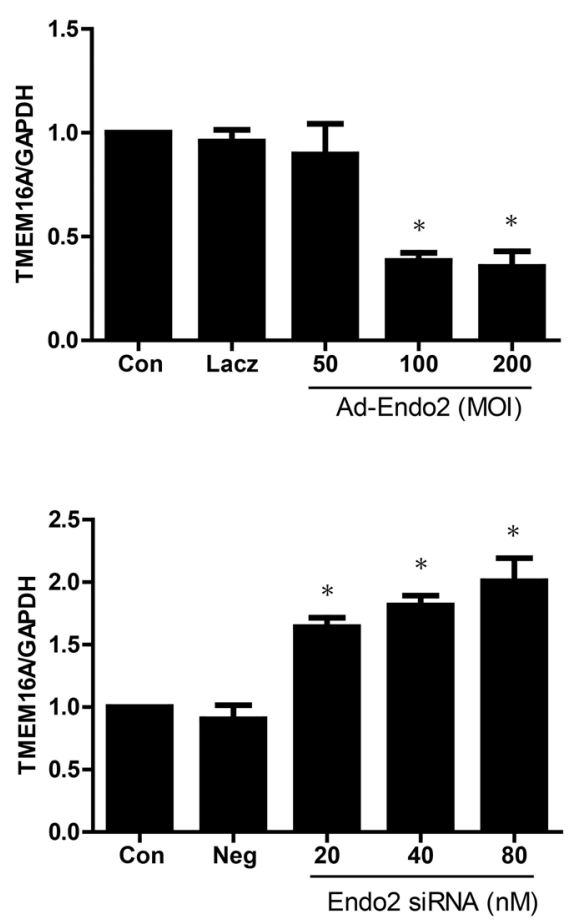

d
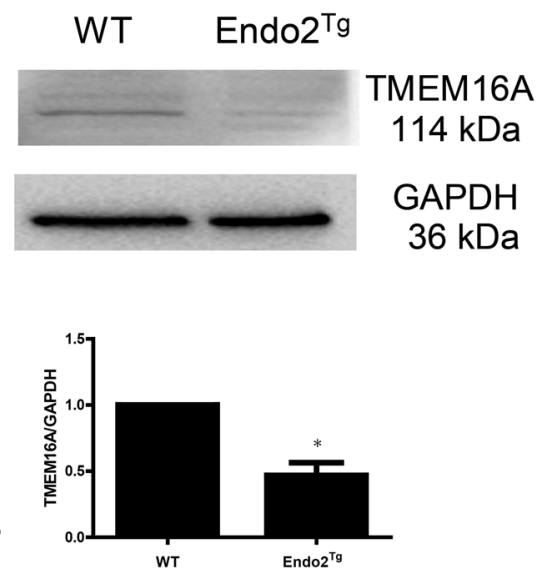

Fig. 3 Endophilin A2 regulates TMEM16A protein expression. a Endophilin A2 had no effects on TMEM16A mRNA level under overexpression (left) or knockdown (right) of Endophilin A2 in BASMCs. b Western blotting showed overexpression (up) of Endophilin A2 decreased TMEM16A expression, whereas knockdown (down) of Endophilin A2 increased TMEM16A expression in BASMCs. Multiplicity of infection (MOI): the number of infected viral particles per cell. $n=6,{ }^{*} P<0.05$ vs. Con. c Representative traces of calcium-activated $\mathrm{Cl}^{-}$current, $I-V$ curves, and mean current densities measured at $\pm 100 \mathrm{mV}$ in BASMCs isolated from wild-type (WT) and Endophilin A2 transgenic mouse $\left(\right.$ endo $2^{\top \mathrm{g}}$ ). $n=8,{ }^{*} P<0.05$ vs. WT. d Expression of TMEM16A in basilar artery from WT and Endophilin A2 transgenic mouse (endo ${ }^{\mathrm{Tg}}{ }^{\text {) }} . n=6$, ${ }^{*} P<0.05$ vs. WT 

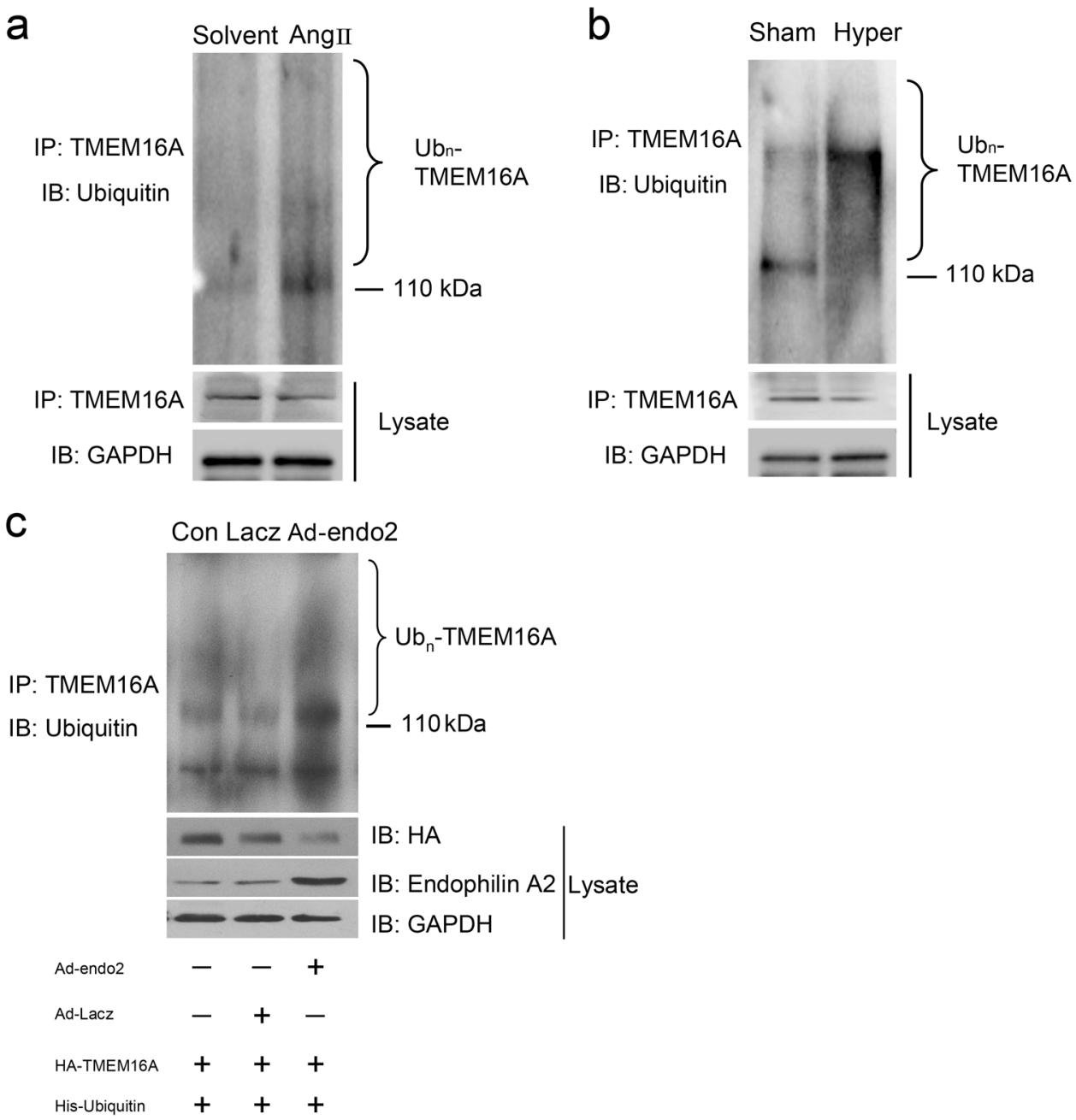

Fig. 4 Involvement of TMEM16A ubiquitination in TMEM16A protein quality control. a BASMCs were treated with Angiotensin II (500 $\mathrm{nM}$ ) for $48 \mathrm{~h}$, then ubiquitination of TMEM16A was detected by Western blotting. b TMEM16A ubiquitination in basilar artery isolated from sham operation (Sham) and hypertensive (Hyper) rats. c Overexpression Endophilin A2 increased TMEM16A ubiquitination in BASMCs. Cells were transfected with HA-TMEM16A and His-ubiquitin plasmids. Then, the cells were infected with Lacz or Endophilin A2 adenovirus as indicated, ubiquitination of TMEM16A was detected by Western blotting

autophagosomes/lysosomes for degradation, which results in a decrease in TMEM16a protein expression and CaCC activity in SMCs.

\section{DISCUSSION}

Our previous study demonstrated that TMEM16A played a significant role in vascular remodeling by inhibiting angiotensin II-induced proliferation in rat basilar smooth muscle cells. The activation of $\mathrm{CaCC}$ and expression of TMEM16A protein were negatively correlated with a medial cross-sectional area in the basilar artery during hypertension [10]. However, how TMEM16A channel activity is negatively regulated during hypertensioninduced remodeling is still unclear. In this study, we found that endophilin A2 was significantly increased in basilar arteries isolated from a $2 \mathrm{k} 2 \mathrm{c}$ renohypertensive rat model and in BASMCs following angiotensin II stimulus, and this result was accompanied by a downregulation of TMEM16A protein expression. Furthermore, by using the whole-cell patch clamp method, we verified that endophilin A2 could modulate $\mathrm{CaCC}$ activity, which implies that endophilin $\mathrm{A} 2$ is an important regulator of $\mathrm{CaCC}$ during hypertension. We also found that overexpression of endophilin A2 largely decreased the expression of TMEM16A protein, whereas its mRNA level did not change. These data suggest that endophilin
A2 regulates the posttranslational modification of TMEM16A protein. Endophilin A2 is a protein with a proline-rich peptide ligand for SH3 domains [21]. Proteins with this functional domain are commonly involved in protein ubiquitination as previously reported [25-27]. Therefore, we speculated that endophilin A2 might play a vital role in TMEM16A ubiquitination during hypertension. The data showed that endophilin A2 undeniably enhanced TMEM16A ubiquitination following angiotensin II stimulus. Although ubiquitination, the process of transferring the target to the proteasome where most cytosolic and nuclear proteins are degraded, is the hallmark of protein degradation, the ability of the proteasome is restrictive due to its inability to degrade most membrane and extracellular proteins, especially oligomeric and aggregated proteins taken up by endocytosis $[28,29]$. TMEM16A is also known to be an oligomeric membrane protein complex similar to many other ion channels, which raises the view that the formation of high-weight TMEM16A oligomeric complexes in the membrane might not result in sufficient degradation in the proteasome [30-32]. Therefore, we hypothesized that the modification of TMEM16A ubiquitination could be degraded through ubiquitin-mediated selective autophagy. By using concanamycin $A$ and bafilomycin $A 1$, our results revealed that autophagosome inhibitors reversed the downregulation effect of TMEM16A mediated by endophilin A2. Moreover, 
a

Concanamycin A (100 nM)

Con Lacz Ad-endo2 Con Lacz Ad-endo2

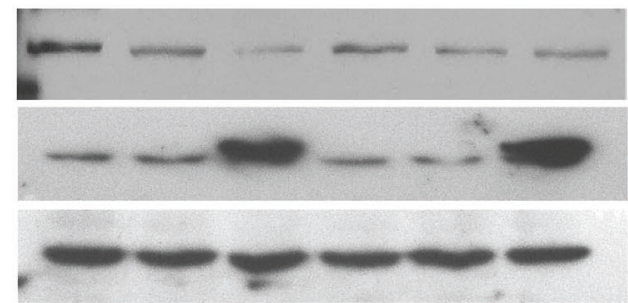

(114 kDa)

Endophilin A2

(47 kDa)

GAPDH

(36 kDa)

b

Bafilomycin A1 (100 nM)

Con Lacz Ad-endo2 Con Lacz Ad-endo2

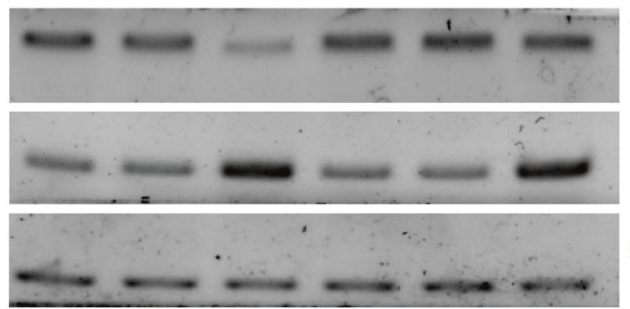

TMEM16A

(114 kDa)

Endophilin A2

(47 kDa)

GAPDH

(36 kDa)
TMEM16A
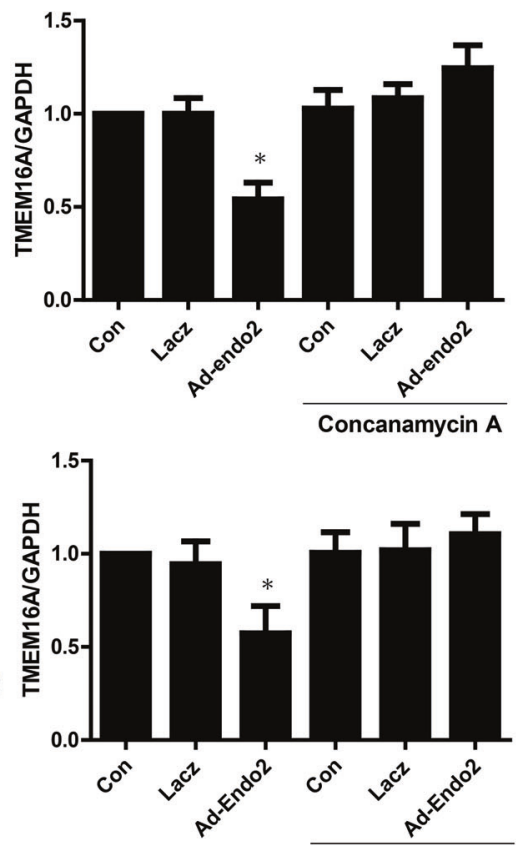

Bafilomycin A1

C

GFP-LC3

Vector+GFP-LC3 RFP-endo2+GFP-LC3
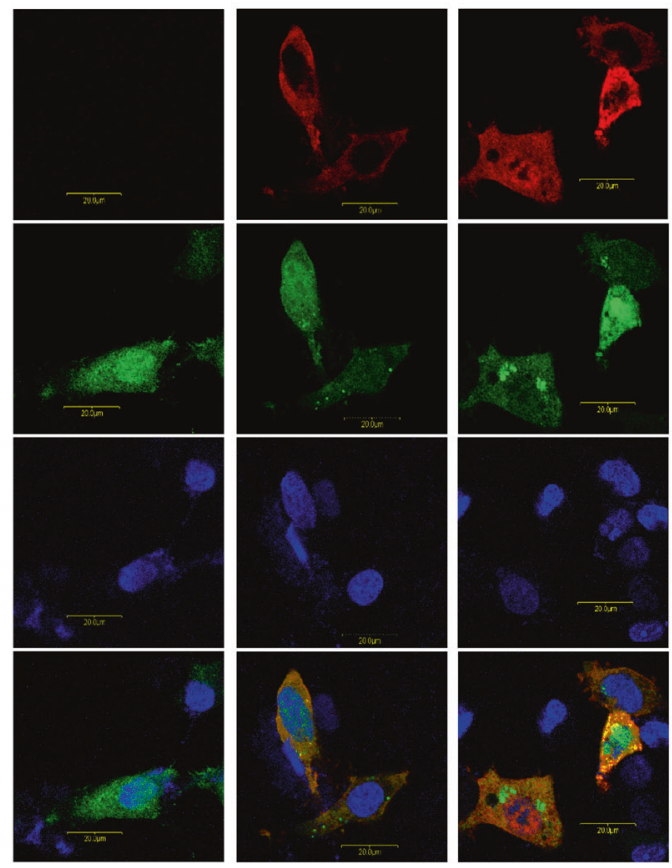

RFP

d Con Lacz Ad-endo2
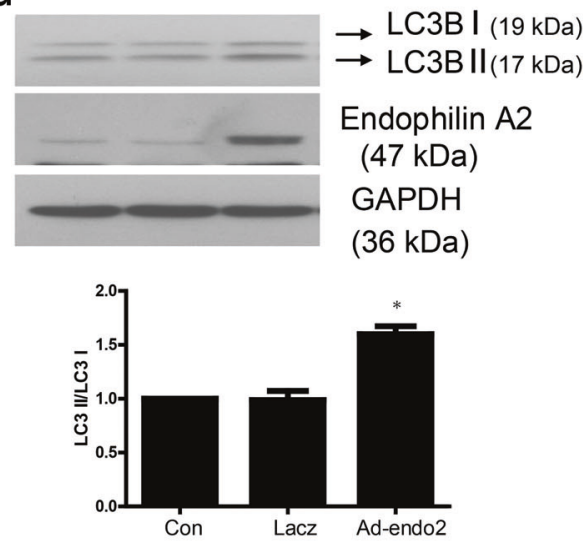

Hoechst

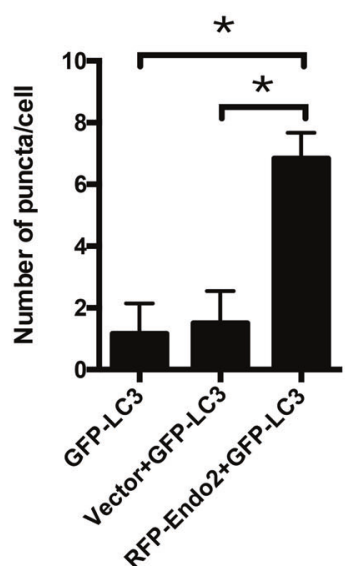

Fig. 5 Autophagosome/lysosome inhibitors attenuate degradation of TMEM16A regulated by Endophilin A2. a Effects of concanamycin A $(100 \mathrm{nM})$ on TMEM16A expression modulated by Endophilin A2. $n=6,{ }^{*} P<0.05$ vs. Con and Ad-Endo2 plus concanamycin A. b Effects of Bafilomycin A1 (100 nM) on TMEM16A expression modulated by Endophilin A2. $n=6,{ }^{*} P<0.05$ vs. Con and Ad-Endo2 plus Bafilomycin A1. c Cells cotransfected with RFP-Endophilin A2 and GFP-LC3 plasmids revealed more significant LC3 puncta in comparison with RFP vector and GFP-LC3 group. Statistically significant differences with $P$-value $<0.05\left(^{*}\right)$. d Overexpression of Endophilin A2 increased the expression of LC 3 and enhanced conversion of LC3 I to LC3 II. $n=6,{ }^{*} P<0.05$ vs. Con 


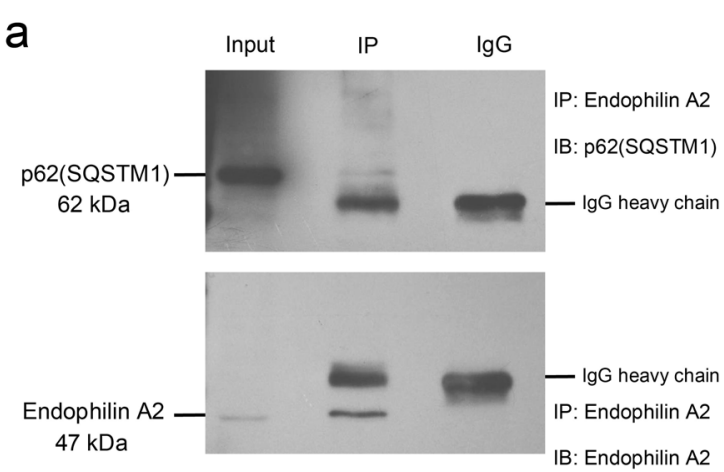
Con Ad-Lacz Ad-Endo2 $\frac{\text { Con Ad-Lacz Ad-Endo2 }}{\text { Con AinA }}$

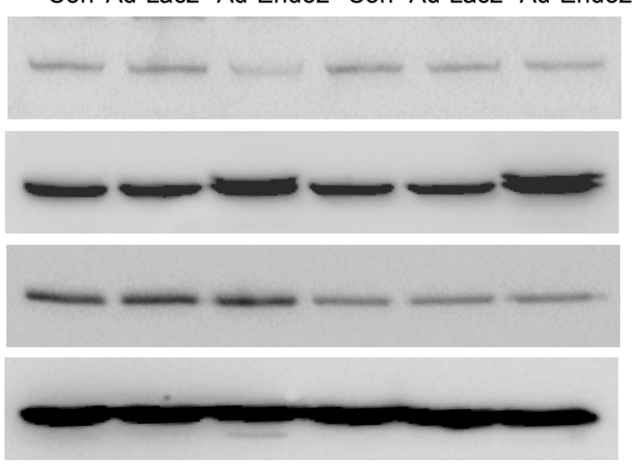

\section{TMEM16A $114 \mathrm{kDa}$ \\ Endophilin A2 $47 \mathrm{kDa}$} p62(SQSTM1) $62 \mathrm{kDa}$

GAPDH $36 \mathrm{kDa}$
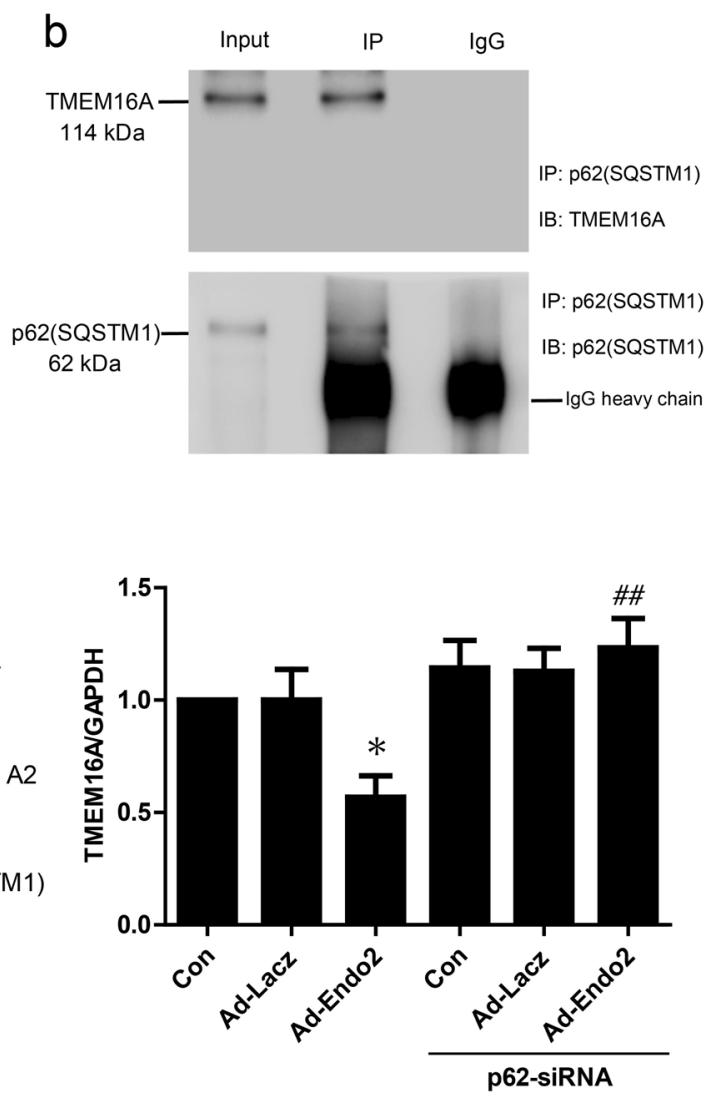

Fig. 6 p62 (SQSTM1) could interact with Endophilin A2 and TMEM16A. a Co-immunoprecipitation analysis of Endophilin A2 and p62 (SQSTM1) protein interaction in BASMCs. b Co-immunoprecipitation analysis of TMEM6A and p62 (SQSTM1) protein interaction in BASMCs. The normal lgG was used as a control. c Effects of p62 knockdown on TMEM16A expression modulated by Endophilin A2. $n=6,{ }^{*} P<0.05$ vs. Con. ${ }^{\# \#} P<0.01$ vs. Ad-Endo2

endophilin $A 2$ increased the formation of autophagosomes and autophagy in BASMCs. Interestingly, it has been reported that endophilin B1 (Bif-1), another member of the endophilin family, is involved in the regulation of autophagy and is required for induced autophagy in Parkinson's disease [33-36]. In our study, we found that endophilin A2 participated in the modulation of autophagy in Vascular Smooth Muscle Cells (VSMCs). However, how endophilin A2 regulates cell autophagy and the following functions in VSMCs requires further study. Furthermore, we demonstrated ubiquitin-binding receptor p62 (SQSTM1) as a critical link that bridged ubiquitinated TMEM16A to autophagosomes [37-40] and that knockdown of p62 (SQSTM1) could reverse the downregulation of TMEM16A modulated by endophi$\operatorname{lin} \mathrm{A} 2$.

Taken together, our studies disclose the associations between endophilin A2 and TMEM16A during hypertension. We found that angiotensin II upregulated endophilin A2 expression and facilitated the inhibition of TMEM16A by ubiquitin-mediated selective degradation, ultimately modulating CaCC activity. Our data provide new insights into the mechanisms by which endophilin $\mathrm{A} 2$ regulates $\mathrm{CaCC}$ activity and angiotensin-II-mediated inhibition of TMEM16A during hypertension-induced vascular remodeling. Future studies are warranted to determine the process of autophagy regulated by endophilin $\mathrm{A} 2$ in hypertension by using genetic models.

\section{ACKNOWLEDGEMENTS}

YYG was supported by the National Natural Science Foundation of China (Key grant numbers 81230082, 81302771, 81525025, 81473206, 81573422,81773721, and
81500226). XFL is supported by the Natural Science Foundation of Guangdong Province (2018A030310233), The Fundamental Research Funds for the Central Universities (number 18zxxt74).

\section{AUTHOR CONTRIBUTIONS}

CZL and FYL conceived and designed the experiments. CZL, FYL, XFL, MMM, XYL, and CXL performed the experiments. CZL and FYL analyzed the data. CZL, FYL, GLW, and YYG wrote the paper.

\section{ADDITIONAL INFORMATION}

The online version of this article (https://doi.org/10.1038/s41401-019-0298-5) contains supplementary material, which is available to authorized users.

Competing interests: The authors declare no competing interests.

\section{REFERENCES}

1. Caputo A, Caci E, Ferrera L, Pedemonte N, Barsanti C, Sondo E, et al. TMEM16A, a membrane protein associated with calcium-dependent chloride channel activity. Science. 2008;322:590-4.

2. Schroeder BC, Cheng T, Jan YN, Jan LY. Expression cloning of TMEM16A as a calcium-activated chloride channel subunit. Cell. 2008;134:1019-29.

3. Yang YD, Cho H, Koo JY, Tak MH, Cho Y, Shim WS, et al. TMEM16A confers receptoractivated calcium-dependent chloride conductance. Nature. 2008;455:1210-5.

4. Pedemonte N, Galietta LJ. Structure and function of TMEM16 proteins (anoctamins). Physiol Rev. 2014;94:419-59.

5. Liu B, Linley JE, Du X, Zhang X, Ooi L, Zhang H, et al. The acute nociceptive signals induced by bradykinin in rat sensory neurons are mediated by inhibition of $M$ type $\mathrm{K}^{+}$channels and activation of $\mathrm{Ca}^{2+}$-activated $\mathrm{Cl}^{-}$channels. J Clin Invest. 2010;120:1240-52. 
6. Cho H, Yang YD, Lee J, Lee B, Kim T, Jang Y, et al. The calcium-activated chloride channel anoctamin 1 acts as a heat sensor in nociceptive neurons. Nat Neurosci. 2012;15:1015-21.

7. Hwang SJ, Blair PJ, Britton FC, O'Driscoll KE, Hennig G, Bayguinov YR, et al. Expression of anoctamin 1/TMEM16A by interstitial cells of Cajal is fundamental for slow wave activity in gastrointestinal muscles. J Physiol. 2009;587:4887-904.

8. Jin $\mathrm{X}$, Shah $\mathrm{S}$, Liu $\mathrm{Y}$, Zhang $\mathrm{H}$, Lees $\mathrm{M}$, Fu Z, et al. Activation of the $\mathrm{Cl}^{-}$channel ANO1 by localized calcium signals in nociceptive sensory neurons requires coupling with the IP3 receptor. Sci Signal. 2013;6:ra73.

9. Huang $F$, Zhang $H$, Wu M, Yang $H$, Kudo $M$, Peters $C J$, et al. Calcium-activated chloride channel TMEM16A modulates mucin secretion and airway smooth muscle contraction. Proc Natl Acad Sci USA. 2012;109:16354-9.

10. Wang $M$, Yang $H$, Zheng LY, Zhang Z, Tang YB, Wang GL, et al. Downregulation of TMEM16A calcium-activated chloride channel contributes to cerebrovascular remodeling during hypertension by promoting basilar smooth muscle cell proliferation. Circulation. 2012;125:697-707.

11. Tien J, Peters $C J$, Wong $X M$, Cheng $T$, Jan YN, Jan LY, et al. A comprehensive search for calcium binding sites critical for TMEM16A calcium-activated chloride channel activity. Elife. 2014;3:e02772.

12. Yang $T$, Hendrickson WA, Colecraft HM. Preassociated apocalmodulin mediates $\mathrm{Ca}^{2+}$-dependent sensitization of activation and inactivation of TMEM16A/16B $\mathrm{Ca}^{2+}$-gated $\mathrm{Cl}^{-}$channels. Proc Natl Acad Sci USA. 2014;111:18213-8.

13. Jung J, Nam JH, Park HW, Oh U, Yoon JH, Lee MG. Dynamic modulation of ANO1/ TMEM16A $\mathrm{HCO}^{-}$permeability by $\mathrm{Ca}^{2+} /$ calmodulin. Proc Natl Acad Sci USA. 2013;110:360-5.

14. Sala-Rabanal M, Yurtsever Z, Nichols CG, Brett TJ. Secreted CLCA1 modulates TMEM16A to activate $\mathrm{Ca}^{2+}$-dependent chloride currents in human cells. Elife. 2015;4:e05875.

15. Perez-Cornejo P, Gokhale A, Duran C, Cui Y, Xiao Q, Hartzell HC, et al. Anoctamin 1 (Tmem 16A) $\mathrm{Ca}^{2+}$-activated chloride channel stoichiometrically interacts with an ezrin-radixin-moesin network. Proc Natl Acad Sci USA. 2012;109:10376-81.

16. Loll PJ, Swain E, Chen Y, Turner BT, Zhang JF. Structure of the SH3 domain of rat endophilin A2. Acta Crystallogr Sect F Struct Biol Cryst Commun. 2008;64:243-6.

17. Gortat A, San-Roman MJ, Vannier C, Schmidt AA. Single point mutation in Bin/ Amphiphysin/Rvs (BAR) sequence of endophilin impairs dimerization, membrane shaping, and Src homology 3 domain-mediated partnership. J Biol Chem. 2012;287:4232-47.

18. Renard HF, Simunovic M, Lemiere J, Boucrot E, Garcia-Castillo MD, Arumugam S, et al. Endophilin-A2 functions in membrane scission in clathrin-independent endocytosis. Nature. 2015;517:493-6.

19. Bai J, Hu Z, Dittman JS, Pym EC, Kaplan JM. Endophilin functions as a membranebending molecule and is delivered to endocytic zones by exocytosis. Cell. 2010;143:430-41.

20. Milosevic I, Giovedi S, Lou X, Raimondi A, Collesi C, Shen H, et al. Recruitment of endophilin to clathrin-coated pit necks is required for efficient vesicle uncoating after fission. Neuron. 2011;72:587-601.

21. Chen Y, Deng L, Maeno-Hikichi Y, Lai M, Chang S, Chen G, et al. Formation of an endophilin- $\mathrm{Ca}^{2+}$ channel complex is critical for clathrin-mediated synaptic vesicle endocytosis. Cell. 2003;115:37-48.

22. Liu CZ, Li XY, Du RH, Gao M, Ma MM, Li FY, et al. Endophilin A2 influences volumeregulated chloride current by mediating $\mathrm{CIC}-3$ trafficking in vascular smooth muscle cells. Circ J. 2016;80:2397-406.
23. Liu YJ, Wang XG, Tang YB, Chen JH, Lv XF, Zhou JG, et al. Simvastatin ameliorates rat cerebrovascular remodeling during hypertension via inhibition of volumeregulated chloride channel. Hypertension. 2010;56:445-52.

24. Ma MM, Lin CX, Liu CZ, Gao M, Sun L, Tang YB, et al. Threonine 532 phosphorylation in $\mathrm{ClC}-3$ is required for angiotensin II-induced $\mathrm{Cl}$ current and migration in cultured vascular smooth muscle cells. $\mathrm{Br} J$ Pharmacol. 2015;173:529-44.

25. Trempe JF, Chen CX, Grenier K, Camacho EM, Kozlov G, McPherson PS, et al. SH3 domains from a subset of BAR proteins define a Ubl-binding domain and implicate parkin in synaptic ubiquitination. Mol Cell. 2009;36:1034-47.

26. Stamenova SD, Dunn R, Adler AS, Hicke L. The Rsp5 ubiquitin ligase binds to and ubiquitinates members of the yeast CIN85-endophilin complex, Sla1-Rvs167. J Biol Chem. 2004;279:16017-25.

27. Khan MM, Strack S, Wild F, Hanashima A, Gasch A, Brohm K, et al. Role of autophagy, SQSTM1, SH3GLB1, and TRIM63 in the turnover of nicotinic acetylcholine receptors. Autophagy. 2014;10:123-36.

28. Kirkin V, Lamark T, Sou YS, Bjorkoy G, Nunn JL, Bruun JA, et al. A role for NBR1 in autophagosomal degradation of ubiquitinated substrates. Mol Cell. 2009;33:505-16.

29. Kraft C, Peter M, Hofmann K. Selective autophagy: ubiquitin-mediated recognition and beyond. Nat Cell Biol. 2010;12:836-41.

30. Tien J, Lee HY, Minor DL Jr, Jan YN, Jan LY. Identification of a dimerization domain in the TMEM16A calcium-activated chloride channel (CaCC). Proc Natl Acad Sci USA. 2013;110:6352-7.

31. Sheridan JT, Worthington EN, Yu K, Gabriel SE, Hartzell HC, Tarran R. Characterization of the oligomeric structure of the $\mathrm{Ca}^{2+}$-activated $\mathrm{Cl}^{-}$channel Ano1/ TMEM16A. J Biol Chem. 2011;286:1381-8.

32. Ohshiro J, Yamamura H, Saeki T, Suzuki Y, Imaizumi Y. The multiple expression of $\mathrm{Ca}^{2+}$-activated $\mathrm{Cl}^{-}$channels via homo- and hetero-dimer formation of TMEM16A splicing variants in murine portal vein. Biochem Biophys Res Commun. 2014:443:518-23.

33. Takahashi Y, Coppola D, Matsushita N, Cualing HD, Sun M, Sato Y, et al. Bif-1 interacts with Beclin 1 through UVRAG and regulates autophagy and tumorigenesis. Nat Cell Biol. 2007;9:1142-51.

34. Takahashi $\mathrm{Y}$, Meyerkord CL, Wang HG. BARgaining membranes for autophagosome formation: regulation of autophagy and tumorigenesis by Bif-1/Endophilin B1. Autophagy. 2008;4:121-4.

35. Takahashi Y, Meyerkord CL, Wang HG. Bif-1/endophilin B1: a candidate for crescent driving force in autophagy. Cell Death Differ. 2009;16:947-55.

36. Wong AS, Lee RH, Cheung AY, Yeung PK, Chung SK, Cheung ZH, et al. Cdk5mediated phosphorylation of endophilin $B 1$ is required for induced autophagy in models of Parkinson's disease. Nat Cell Biol. 2011;13:568-79.

37. Moscat J, Diaz-Meco MT, Wooten MW. Signal integration and diversification through the p62 scaffold protein. Trends Biochem Sci. 2007;32:95-100.

38. Komatsu M, Waguri S, Koike M, Sou YS, Ueno T, Hara T, et al. Homeostatic levels of p62 control cytoplasmic inclusion body formation in autophagy-deficient mice. Cell. 2007;131:1149-63.

39. Kirkin V, Dikic I. Role of ubiquitin- and Ubl-binding proteins in cell signaling. Curr Opin Cell Biol. 2007;19:199-205.

40. Pankiv S, Clausen TH, Lamark T, Brech A, Bruun JA, Outzen $H$, et al. p62/SQSTM1 binds directly to Atg8/LC3 to facilitate degradation of ubiquitinated protein aggregates by autophagy. J Biol Chem. 2007;282:24131-45. 